\title{
OPEN Kinetics of slow release of nitrogen fertiliser from multi-layered nanofibrous structures
}

\author{
Leila Javazmi ${ }^{1,2 \bowtie}$, Anthony Young ${ }^{2,3}$, Gavin J. Ash $\oplus^{2}$ \& Tobias Low ${ }^{1}$ \\ Fertilisers are essential in modern agriculture to enhance plant growth, crop production and product \\ quality. Recent research has focused on the development of delivery systems designed to prolong \\ fertiliser release. This study introduces a new technology to encapsulate and release molecules of \\ fertilisers by using multi-layered electrospun nanofibre as a carrier. Single-layer poly L-lactic acid \\ (PLLA) nanofibres loaded with urea were fabricated using electrospinning. Triple-layer nanofibrous \\ structures were produced by electrospinning polyhydroxybutyrate (PHB) nanofibres as external \\ layers with PLLA nanofibres impregnated with urea fertiliser as the middle layer. Scanning electron \\ microscopy (SEM) and Fourier transform infrared spectrophotometry (FTIR) were employed to \\ characterize the morphology of electrospun nanofibres. Urea release dynamic was analysed using \\ a total nitrogen instrument (TNM-1). The results indicated that triple-layered urea-impregnated \\ nanofibrous structures led to lower initial rate of nitrogen release and slower release rate of \\ cumulative nitrogen which extended for more than three months. It is concluded that triple-layer \\ nanofibrous structures have the potential for slow release delivery of fertilisers.
}

Nanofibres are filaments with diameters in the nanometre range and an aspect ratio (length: diameter) larger than $100: 1^{1,2}$. This intrinsic features of nanofibres increases the surface area to volume ratio and decreases spaces between individual nanofibres compared to regular fibres ${ }^{3,4}$. There are several methods used to prepare nanofibres, encompassing both top-down (melt-blown, melt electrospinning, islands-in-the-sea, and electrospinning) and bottom-up (interfacial polymerization, self-assembly, and phase separation) approaches ${ }^{5}$.

Electrospinning is a production technique to prepare ultrafine fibres at a micro- or nanoscale. It has the ability to spin a broad range of polymers, the potential for upscale, and provides precise control over morphology, aspect ratio, pore-size distribution and porosity in comparison to other approaches to forming continuous nanofibres ${ }^{6,7}$. Furthermore, electrospun nanofibres are ideal porous membranes given their open-pore structure (tens of nanometres to one micrometre), high surface area and high gas permeability. Such nanofibres have been used in areas as diverse as filtrations, nanocomposites, drug delivery, biomedical, medical prostheses, fuel cells, sensors, and protective clothing ${ }^{5,8}$. However, the use of electrospun nanofibers in agriculture is novel and still in its infancy ${ }^{9}$.

Fertilisers are used to promote plant growth, increase crop production and improve quality of products. The officious use of nitrogenous fertiliser is hindered by system losses occurring through volatilization and leaching ${ }^{10}$. Recent investigations have focused on the development of systems using different materials that prolong the release of fertilisers ${ }^{11}$. These systems aim to minimise soil contamination by controlling fertiliser release using low cost sustainable materials ${ }^{10}$. Electrospun nanofibres have a very high specific surface area which makes them promising candidates for delivery of agrichemicals ${ }^{12-14}$. Electrospun nanofibres have an advantage over particulate carriers to control fertiliser encapsulation as they are less likely to be washed away than nanoparticles ${ }^{9,15}$. As a result, agricultural producers can potentially decrease the amount of fertiliser loss and prevent potential environmental contamination, as well as fertiliser run-off, by using nanofibrous networks ${ }^{15}$.

Urea is a cost-effective solid nitrogen-based fertiliser used to promote plant growth and increase crop production. However, uncontrolled excessive use of urea fertiliser is harmful to plants and can lead to soil and water pollution ${ }^{12}$. Thus, significant research efforts have focused on prolonged-released systems that minimise adverse environmental impacts and increase the efficiency of urea fertiliser use ${ }^{16}$.

Electrospun nanofibres have been demonstrated as potential vehicles for agrichemical delivery. Urea impregnated onto wheat gluten electrospun-fibre membranes was found to have a rapid release in the first $10 \mathrm{~min}$,

\footnotetext{
${ }^{1}$ School of Mechanical and Mechatronic Engineering, University of Southern Queensland, Toowoomba, Australia. ${ }^{2}$ Centre for Crop Health, University of Southern Queensland, Toowoomba, Australia. ${ }^{3}$ School of Agriculture and Food Sciences, The University of Queensland, Saint Lucia, Australia. ${ }^{\bowtie}$ email: Leila.javazmi@ usq.edu.au
} 
followed by a decreased rate until equilibrium at $5 \mathrm{~h}$, when $98 \%$ was released ${ }^{16}$. While demonstrating the potential for nanofibre delivery of agrichemicals, the release dynamics were not suitable for slow-release fertilisers ${ }^{17}$. An advancement was coaxial electrospinning where urea was incorporated in the PLLA core surrounded by a PHB sheath. This released urea for a month and biodegraded within three months ${ }^{18}$. To the best of our knowledge, no research has reported encapsulation and release of agricultural chemicals from triple-layered nanofibrous structures. The focus of this study was to provide proof of concept of this novel approach to the encapsulation of urea within a triple-layered nanofibrous matrix.

\section{Results and discussion}

Electrospinning of single-layered and triple-layered nanofibre mats loaded with urea. A custom electrospinning apparatus was used to produce single-layered PLLA nanofibres loaded with different concentrations of urea. The PLLA solution was fixed at $5 \%(\mathrm{w} / \mathrm{w})$, while urea was loaded at $10 \%, 20 \%$, and $40 \%$ $(\mathrm{w} / \mathrm{w})$, relative to the mass amount of PLLA, and transferred to a $1 \mathrm{~mL}$ syringe with an attached 18-gauge blunt tip needle. Single-layered PLLA nanofibres loaded with different urea concentrations were collected onto an aluminium foil surface and stored in a desiccator under vacuum for $24 \mathrm{~h}$ prior to use.

Triple-layer nanofibre mats consisting of a layer of PLLA sandwiched between PHB layers were prepared. A PHB polymeric solution with concentration of $7 \%(\mathrm{w} / \mathrm{w})$ was fed into the electrospinning apparatus using a $1 \mathrm{~mL}$ syringe to produce the first PHB nanofibre layer on the surface of aluminium foil collector. Subsequently, 5\% PLLA solution with 5\%,10\%,20\% and 40\% (w/w) urea was electrospun onto the PHB nanofibre layer. Finally, the outer surface of PLLA nanofibre layer was electrospun by another layer of PHB nanofibre resulting in a PHB/ PLLA/PHB triple-layer nanofibrous structure.

Nanofibre characterization. SEM (FEI Quanta 200 SEM 2002) at the Royal Melbourne Institute of Technology (RMIT) Microscopy and Microanalysis Facility (RMMF) and benchtop SEM (JEOL JCM-6000PLUS) at the University of Southern Queensland (USQ) were used to determine the morphology of electrospun nanofibres. Image J processing software was used to measure PLLA and PHB nanofibre diameters from high magnification SEM images.

The morphology of electrospun nanofibres is dependent on electrospinning parameters including applied voltage, solution composition, solution concentration, solution feed rate and collection distance ${ }^{19}$. When the concentration of a polymeric solution increases, the solution viscosity increases ${ }^{20}$. By keeping all electrospinning parameters constant, and increasing the concentration of a polymeric solution, nanofibres are formed thicker as the higher viscosity solution which prevents the polymer jet from stretching further and electrospinning thinner fibres ${ }^{21}$.

In this study, the effect of urea concentration on nanofibre morphology was investigated. The diameter of PLLA electrospun nanofibres increased from 496 to $782 \mathrm{~nm}$ as urea concentration increased from 0 to $40 \%$ (Fig. 1, Table 1). The morphology and diameter distribution of 7\% PHB electrospun nanofibres are shown in Fig. 1I,J. The average diameters of the outer layer PHB nanofibres were $418 \pm 64 \mathrm{~nm}$. A typical SEM micrograph cross section of triple-layer PHB/PLLA/PHB containing 10\% urea is illustrated in Fig. 1K,L. The thickness of the triple-layer nanofibrous structure is approximately $21 \mu \mathrm{m}$. The micrograph illustrates the nanofibre arrangement and void spaces in the triple-layered structure.

Brunauer-Emmett-Teller (BET) analysis of the 5\% PLLA electrospun nanofibres without loading urea indicated that the porous fibrous mat was fabricated with a surface area of $188 \mathrm{~m}^{2} / \mathrm{g}$, a mean pore diameter of $37 \mathrm{~nm}$ and a total pore area of $10 \mathrm{~m}^{2} / \mathrm{g}$. Thus, the structure satisfies the definition for nanofibrous structures ${ }^{22}$.

The FTIR spectrograph of urea powder and single- and triple-layered PLLA nanofibres loaded with 10\%, $20 \%$ and $40 \%$ urea is shown in Fig. 2. Characteristic peaks of PLLA form at 1090-1190 $\mathrm{cm}^{-1}$ (ester bond), a middle peak at $1362 \mathrm{~cm}^{-1}$ (-CH3 symmetric bending vibration), and two peaks at 2997 and $2947 \mathrm{~cm}^{-1}$ (methyl groups) ${ }^{23}$. A characteristic strong peak of PLLA at about $1750 \mathrm{~cm}^{-1}$ is related to the stretching vibration of the carbonyl group $(\mathrm{C}=\mathrm{O})$ shifted slightly from $1755 \mathrm{~cm}^{-124}$. In the FTIR spectra of urea, the peak in the region of $1629-1680 \mathrm{~cm}^{-1}$ is due to the $\mathrm{C}=\mathrm{O}$ stretching bond of urea and the presence of absorption peaks at 3344,3447 and $1157 \mathrm{~cm}^{-1}$ can be associated to the stretching vibration of $\mathrm{N}-\mathrm{H}$ bonds ${ }^{25}$. The characteristic peaks of urea at $1458 \mathrm{~cm}^{-1}$ and approximately $1596 \mathrm{~cm}^{-1}$ reflect the stretching vibration of the $\mathrm{C}-\mathrm{N}$ bond and $\mathrm{N}-\mathrm{H}$ bonding, respectively ${ }^{26}$. The urea characteristic peaks at $1458 \mathrm{~cm}^{-1}(\mathrm{C}-\mathrm{N}$ bond $), 1157 \mathrm{~cm}^{-1}(\mathrm{~N}-\mathrm{H}$ bond) and between 1629-1680 $\mathrm{cm}^{-1}(\mathrm{C}=\mathrm{O}$ stretching) are all present in the FTIR spectrums of single- and triple-layered nanofibrous structures. However, peak positions of $\mathrm{C}=\mathrm{O}$ and $\mathrm{N}-\mathrm{H}$ bonds in PLLA nanofibres have been shifted due to changes in vibrational frequency which can occur through changes in bond strength or in reduced mass of urea ${ }^{27}$.

Urea release characteristics. A TNM-1 total nitrogen instrument (Shimadzu) was used to determine the cumulative percentage of nitrogen released from single- and triple-layer nanofibres containing 10\%, 20\%, and $40 \%$ urea, respectively (Table 2). Increasing urea percentage from $10 \%$ to $40 \%$ in both single-layered PLLA nanofibre mat, and triple-layered PHB/PLLA/PHB nanofibrous structures, resulted in initial nitrogen release at a higher rate $(P$ value $=0.000)$ (Table 2$)$. The nitrogen release rate of single-layered nanofibres was not significantly affected by various urea concentration $(P$ value $=0.361)($ Fig. $3 \mathrm{~A})$, however, increasing urea concentration into triple-layered nanofibres resulted in significantly higher nitrogen release rate $(P$ value $=0.000)$. Statistical analysis of the results (using a one-way ANOVA) for cumulative nitrogen release showed that at different urea concentrations, triple-layered nanofibres containing $10 \%$ urea showed a highly significant decrease in release rate compared with other samples $(P$ value $=0.000)$.

For PLLA impregnated with $10 \%$ w/w urea, after 39 hours the triple-layered nanofibre mats had released less than $50 \%$, while the single layer mats had released over $80 \%$. Based on these results, the experiment was repeated 

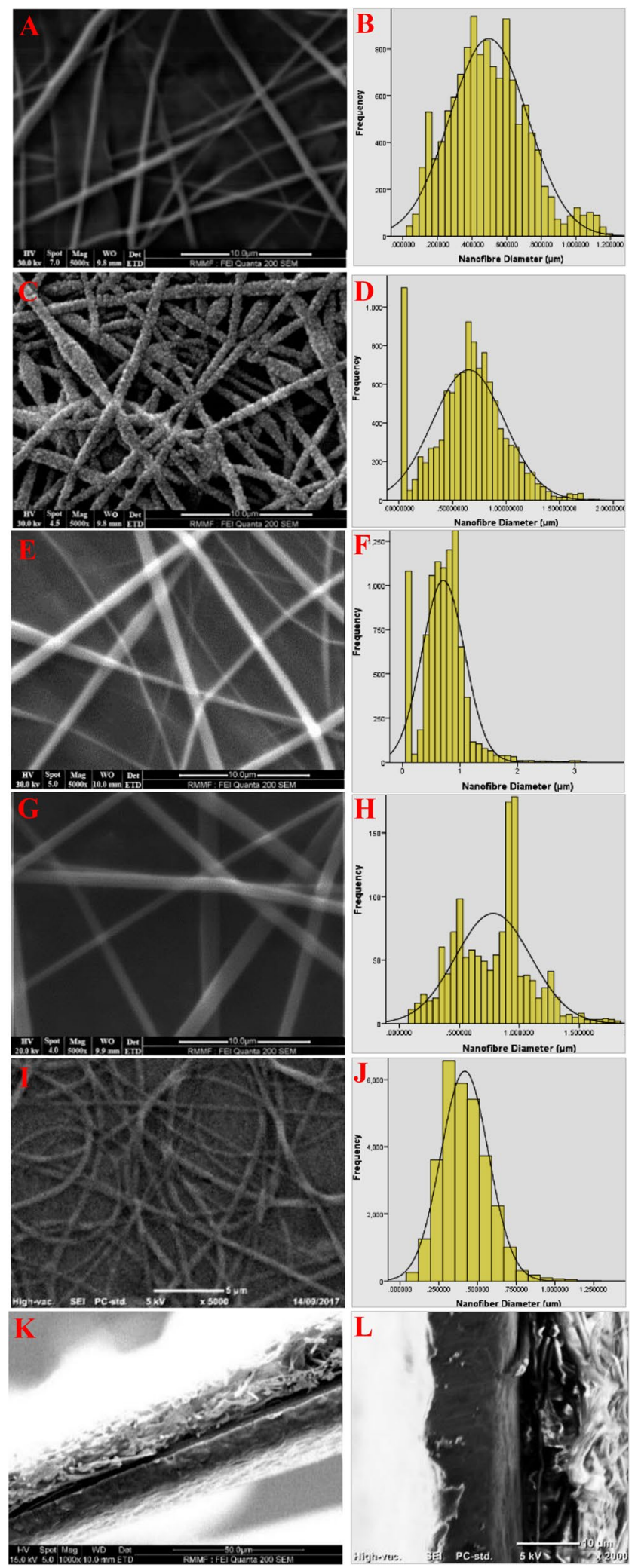

Figure 1. SEM images and related diameter histograms of PLLA electrospun nanofibres containing urea at concentrations of $0 \%{ }^{\star}(\mathbf{A}, \mathbf{B}), 10 \%{ }^{* *}(\mathbf{C}, \mathbf{D}), 20 \%{ }^{\star}(\mathbf{E}, \mathbf{F})$, and $40 \%{ }^{\star}(\mathbf{G}, \mathbf{H})$. SEM images and diameter distribution of $7 \%$ PHB electrospun nanofibres (I,J). SEM image of cross section of PHB/PLLA/PHB triple-layer nanofibrous structure containing $10 \%$ urea at $(\mathbf{K}) 1000 \times$ magnification, and $(\mathbf{L}) 2000 \times$ magnification. ${ }^{*}$ Gold coating ${ }^{* *}$ Carbon coating. 


\begin{tabular}{|l|l|l|}
\hline Urea concentration $(* \% \mathbf{w} / \mathbf{w})$ & Nanofibre diameter $(\mathbf{n m})$ & ${ }^{* *} \mathbf{C V} \%$ \\
\hline 0 & 496.183 & $22 \%$ \\
\hline 10 & 650.154 & $33 \%$ \\
\hline 20 & 710.000 & $37 \%$ \\
\hline 40 & 782.231 & $31 \%$ \\
\hline
\end{tabular}

Table 1. Effect of urea concentration on electrospun nanofibre diameter. ${ }^{\star} \% \mathrm{w} / \mathrm{w}$ based on amount of PLLA used. ${ }^{*}$ Coefficient of Variation $(\mathrm{CV})$.

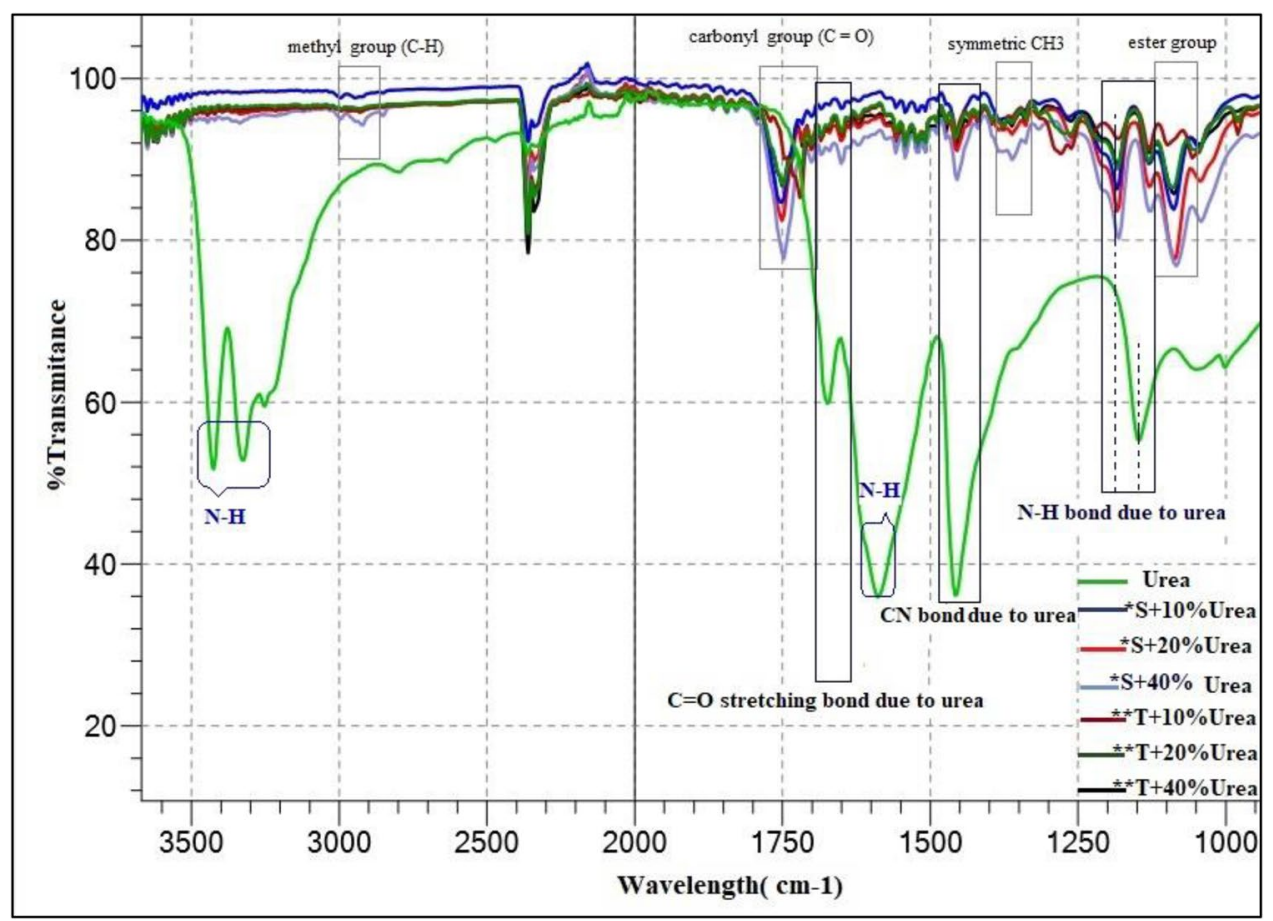

Figure 2. FTIR spectra of urea and single-, triple-layered PLLA nanofibres loaded with urea. Pure urea (Urea), PLLA nanofibre containing $10 \%$ urea $\left({ }^{\star} \mathrm{S}+10 \%\right.$ Urea), PLLA nanofibre containing $20 \%$ urea $\left({ }^{\star} \mathrm{S}+20 \%\right.$ Urea), PLLA nanofibre containing $40 \%$ urea $\left({ }^{*} \mathrm{~S}+40 \%\right.$ Urea), PHB/PLLA/PHB nanofibre containing $10 \%$ urea $\left({ }^{*} \mathrm{~T}+\right.$ $10 \%$ Urea), PHB/PLLA/PHB nanofibre containing $20 \%$ urea $\left({ }^{*} \mathrm{~T}+20 \%\right.$ Urea), PHB/PLLA/PHB nanofibre containing $40 \%$ urea $\left({ }^{\star *} \mathrm{~T}+40 \% \mathrm{Urea}\right) .{ }^{\star} \mathrm{S}$ : Single-layered PLLA nanofibre mat, ${ }^{* *} \mathrm{~T}$ : Triple-layered PHB/PLLA/ PHB nanofibrous structure.

for single- and triple-layered nanofibrous structure containing $10 \%$ urea. Figure 3B shows cumulative nitrogen release from single- and triple-layered nanofibres loaded with $10 \%$ urea as well as the control sample for the first 12 hours. Results revealed that triple-layer nanofibrous structure containing $10 \%$ urea released nitrogen at a slower rate compared to single-layer nanofibrous structure encapsulating urea by either electrospinning fabrication or immersing deposition. The physical barrier of PHB nanofibre layers coating PLLA nanofibre layer resulted in slower rate of urea release in triple-layered nanofibre containing $10 \%$ urea compared to single-layered PLLA nanofibre. Furthermore, increasing the urea content into single- and triple-layered PLLA nanofibres resulted in increasing bead numbers in samples ${ }^{28}$. Thus, fabricated multi-layered nanofibres can be considered a means of delaying urea release from a PLLA nanofibrous matrix.

\section{Conclusion}

Single- and triple-layer nanofibrous structure were fabricated and loaded with urea using a custom-built electrospinning device. Fourier transform infrared Spectrophotometer analysis showed that urea was encapsulated in all samples. As expected, increased urea concentration led to larger nanofibre diameters. Increasing the percentage of urea loaded into nanofibres from $10 \%$ to $40 \%$ increased the initial rate of nitrogen release. Both single- and triple- layered nanofibres samples released nitrogen for three months. Although high urea concentrations did not affect the nitrogen release rate for single-layer nanofibres significantly ( $P$ value $=0.361$ ), increasing urea content into triple-layered nanofibres resulted in increasing nitrogen release content significantly ( $P$ value $=0.000)$. Triple-layer nanofibrous structures containing $10 \%$ urea exhibited a significantly lower level and lower initial rate of nitrogen release compared to single-layer nanofibres loading $10 \%$ urea $(P$ value $=0.012)$. In conclusion, 


\begin{tabular}{|l|l|l|l|l|l|l|}
\hline Time (h)/sample & S10\% urea & S20\% urea & S40\% urea & T10\% urea & T20\% urea & T40\% urea \\
\hline 1 & $27.30 \%$ & $62.00 \%$ & $78.60 \%$ & $23.30 \%$ & $50.80 \%$ & $69.50 \%$ \\
\hline 2 & $59.10 \%$ & $70.70 \%$ & $81.60 \%$ & $32.70 \%$ & $64.60 \%$ & $79.00 \%$ \\
\hline 4 & $75.20 \%$ & $78.30 \%$ & $82.80 \%$ & $38.50 \%$ & $71.80 \%$ & $81.70 \%$ \\
\hline 14 & $79.70 \%$ & $87.60 \%$ & $85.30 \%$ & $38.50 \%$ & $83.20 \%$ & $85.10 \%$ \\
\hline 39 & $84.80 \%$ & $91.60 \%$ & $88.90 \%$ & $45.90 \%$ & $88.40 \%$ & $90.30 \%$ \\
\hline 63 & $89.00 \%$ & $93.00 \%$ & $91.00 \%$ & $50.30 \%$ & $90.40 \%$ & $91.30 \%$ \\
\hline 87 & $90.60 \%$ & $93.50 \%$ & $91.20 \%$ & $60.40 \%$ & $91.00 \%$ & $91.50 \%$ \\
\hline 137 & $90.60 \%$ & $95.00 \%$ & $91.40 \%$ & $67.80 \%$ & $91.60 \%$ & $93.00 \%$ \\
\hline 230.5 & $91.80 \%$ & $96.00 \%$ & $92.50 \%$ & $67.80 \%$ & $93.00 \%$ & $93.30 \%$ \\
\hline 278.5 & $91.80 \%$ & $95.90 \%$ & $92.80 \%$ & $67.80 \%$ & $93.40 \%$ & $93.30 \%$ \\
\hline 1570.5 & $96.10 \%$ & $97.60 \%$ & $95.80 \%$ & $82.50 \%$ & $96.80 \%$ & $95.90 \%$ \\
\hline 1592 & $96.10 \%$ & $97.60 \%$ & $96.20 \%$ & $82.50 \%$ & $96.80 \%$ & $95.90 \%$ \\
\hline 1735.5 & $97.20 \%$ & $98.20 \%$ & $96.70 \%$ & $87.40 \%$ & $97.40 \%$ & $96.80 \%$ \\
\hline 2963 & $100.00 \%$ & $100.00 \%$ & $100.00 \%$ & $100.00 \%$ & $100.00 \%$ & $100.00 \%$ \\
\hline 3000 & $100.00 \%$ & $100.00 \%$ & $100.00 \%$ & $100.00 \%$ & $100.00 \%$ & $100.00 \%$ \\
\hline$P$ value for initial nitrogen release & 0.000 & & & 0.000 & & \\
\hline
\end{tabular}

Table 2. Nitrogen release rate from single- and triple-layer nanofibre structures from first hour till $3000 \mathrm{~h}$. $S$ single-layered PLLA nanofibre mat, $T$ triple-layered PHB/PLLA/PHB nanofibrous structure, $P$ value probability value.

triple-layered electrospun PLLA nanofibres containing urea may be an effective carrier to control the release of urea fertiliser in agriculture applications. These materials have the potential as slow-release delivery systems for high-value agrochemicals.

\section{Methods}

Materials. Poly (L-lactide) (PLLA) with a molecular weight of $282 \mathrm{kD}$ was purchased from Vorina Biomaterials Company in Ireland (CAS Number: 33135-50-1). Poly [(R)-3-hydroxybutyric acid] (PHB) was provided from Sigma Aldrich (Product Number: 363502). Solvents, N-Dimethylformamide (DMF); Reagent Plus ${ }^{\oplus}, \geq 99 \%$, chloroform (CF); anhydrous, $\geq 99 \%$. Acetone (AC) for HPLC, $\geq 99.8 \%$ were obtained from Australia Sigma Aldrich. Urea (N: P: K; 46-0-0) was purchased from Richgro Garden Products. The amount of nitrogen is equal to $46 \%$ urea mass.

Preparation of PLLA and PHB solutions. PLLA solution with optimum concentrations of 5\% (w/w) in chloroform: acetone $(3: 1 \mathrm{v} / \mathrm{v})$ was prepared and mixed with 10, 20, and 40\% (w/w) urea powder relative to the weight of PLLA. Polyhydroxybutyrate polymeric solution in dimethylformamide: chloroform (30:70 v/v) solvents was prepared at concentration of $7 \%(\mathrm{w} / \mathrm{w})$.

Electrospinning apparatus. The schematic setup for nanofibre electrospinning is shown in Fig. 4. It consists of a high voltage power supply, model 73,030, DC input $30 \mathrm{kV} @ 1 \mathrm{~mA}$, (Genvolt, Ireland), and a New Era NE-300 "Just Infusion" syringe pump. A metal frame $14 \mathrm{~cm} \times 16 \mathrm{~cm}$ with attached aluminium foil is located $15 \mathrm{~cm}$ from the syringe needle to collect the nanofibres. The positive terminal of the power supply is connected to the needle and the ground terminal is attached to the collector (metal frame). The electrospinning process occurs between the needle tip and the aluminium collector and nanofibres gather on the surface of the aluminium foil.

Electrospinning was conducted at $32{ }^{\circ} \mathrm{C}$ with $12(+\mathrm{kV})$ high voltage, and $20(+\mathrm{kV})$ high voltage for PLLA and PHB solutions, respectively. Feed rate and needle size for both solutions were selected at $1 \mathrm{~mL} / \mathrm{hr}$ and $18 \mathrm{G}$. The morphology of electrospun nanofibres was determined by varying the urea concentration ranging from $0 \%$ to $40 \%$ in a fixed 5\% PLLA solution.

Characterisation. Accelerated Surface Area and Porosimetry System (ASPA) 2400 Micropore Data Reduction was used to study BET analyses for the 5\% PLLA electrospun nanofibre to evaluate surface areas, pore diameters and total pore area.

FTIR measurements of urea powder and single-, triple-layered PLLA nanofibres loaded with 10\%, 20\% and $40 \%$ urea were carried out using IRAffinity-1S Fourier transform infrared Spectrophotometer (Shimadzu). Aluminium foil was used to place the samples under spectrophotometer.

The single- and triple-layered nanofibrous mats with a thickness of approximately 7 and $21 \mu \mathrm{m}$, respectively, were cut into $2 \mathrm{~cm} \times 6 \mathrm{~cm}$ pieces. These pieces were accurately weighed $\pm 0.00001 \mathrm{~g}$ and placed in a plastic tube with $20 \mathrm{~mL}$ of milli-Q water to soak for 2 minutes to wash out any deposited urea fertiliser on the surface of the nanofibrous mats. Subsequently, each sample was immersed in a $50 \mathrm{~mL}$ plastic tube with $20 \mathrm{~mL}$ milli-Q water and placed in a shaker at $70 \mathrm{RPM}$ and $30^{\circ} \mathrm{C}^{18}$. The samples were removed from the shaker and immersed in the 
A

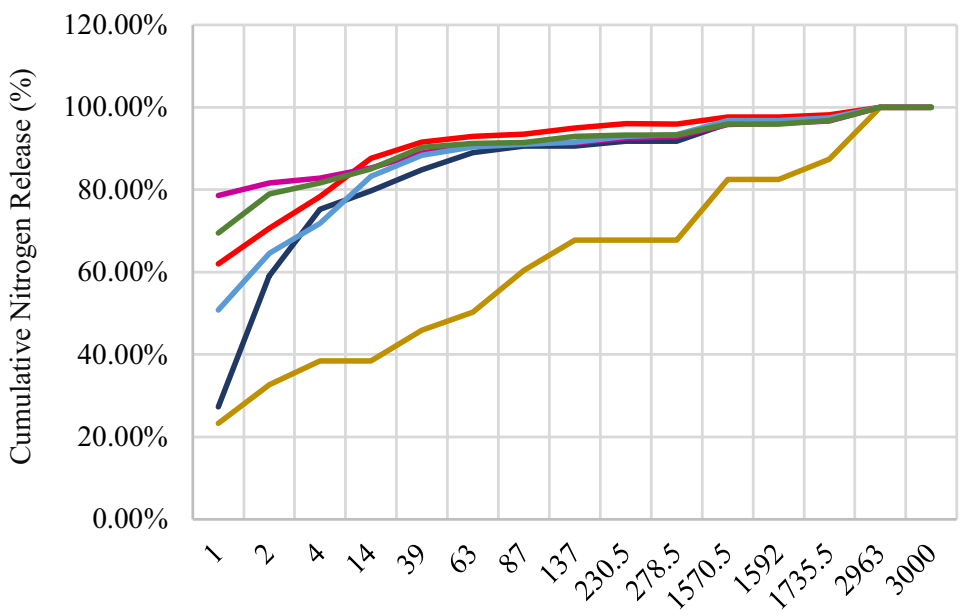

Time (hour)

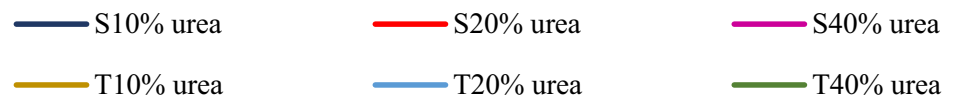

B

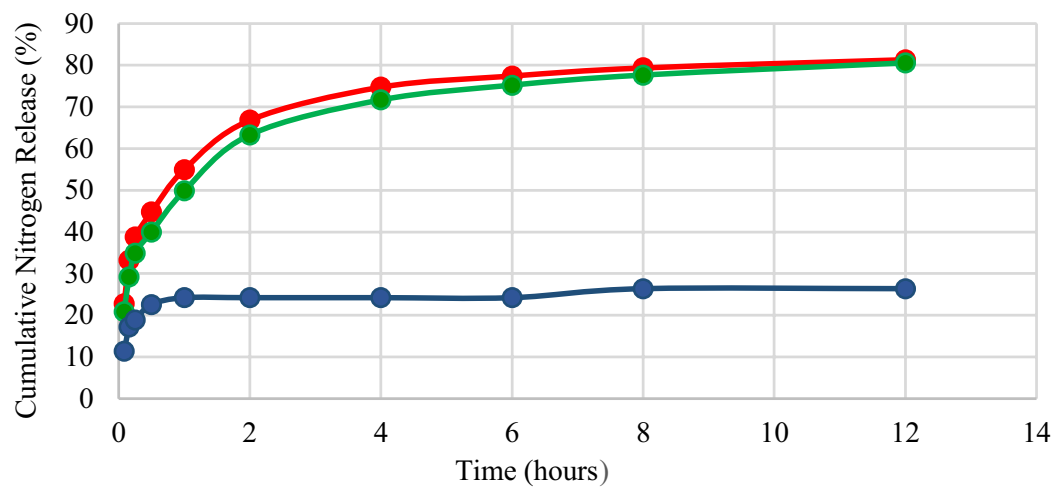

$\longrightarrow$ Nanofibres immersed in urea solution

—- Single layered nanofibre loading $10 \%$ urea

$\longrightarrow$ Triple layered nanofibres loading $10 \%$ urea

Figure 3. (A) Cumulative nitrogen release from single- and triple-layer nanofibre structures from first hour until 3000 hours, and (B) Cumulative nitrogen release from immersed nanofibres in urea solution and single-, triple-layer nanofibrous structure containing 10\% urea from first hour until 12 hours. $S$ single-layered PLLA nanofibre mat, $T$ triple-layered PHB/PLLA/PHB nanofibrous structure.

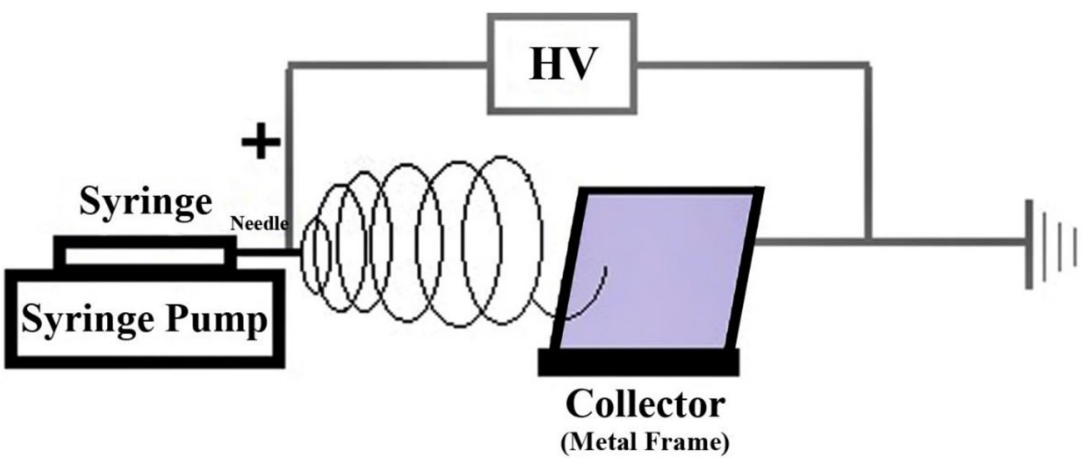

Figure 4. Schematic diagram of the electrospinning apparatus. 
next tube containing $20 \mathrm{~mL}$ milli-Q water at different periods, ranging from 0 up to 3000 hours. A TNM-1 total nitrogen instrument (Shimadzu) was used to measure nitrogen release from the nanofibre mats. The data were reported as percent nitrogen. Cumulative nitrogen release from each sample was calculated for the determined period. A 5\% PLLA nanofibre mat without loading urea immersed in $0.5 \%$ urea solution for 24 hours, was used as the control sample. All experiments were carried out three times and results were reported as average \pm one standard deviation.

Received: 17 April 2019; Accepted: 12 February 2021

Published online: 01 March 2021

\section{References}

1. Kiyak, Y. \& Cakmak, E. Nanofiber production methods. Electron. J. Text. Technol. 8, 49-60 (2014).

2. Huang, Z. M., Zhang, Y. Z., Kotaki, M. \& Ramakrishna, S. A review on polymer nanofibers by electrospinning and their applications in nanocomposites. Compos. Sci. Technol. 63, 2223-2253 (2003).

3. Subbiah, T., Bhat, G. S., Tock, R. W., Parameswaran, S. \& Ramkumar, S. S. Electrospinning of nanofibers. J. Appl. Polym. Sci. 96, 557-569 (2005).

4. Doshi, J. \& Reneker, D. H. Electrospinning process and applications of electrospun fibers. J. Electrost. 35, 151-160 (1993).

5. Ali, U., Zhou, Y., Wang, X. \& Lin, T. Electrospinning of Continuous Nanofiber Bundles and Twisted Nanofiber Yarns. Nanofibers Prod. Prop. Funct. Appl. 153-174 (2011).

6. Ravandi, S. A. H., Tork, R. B., Dabirian, F., Gharehaghaji, A. A. \& Sajjadi, A. Characteristics of yarn and fabric made out of nanofibers. Mater. Sci. Appl. 6, 103-110 (2015).

7. Javazmi, L., Ravandi, S. A. H. \& Ghareaghaji, A. A. Fabrication and characterization of PET nanofiber hollow yarn. Fibers Polym. 15, 954-960 (2014).

8. Basel Bazbouz, M. An Investigation of Yarn Spinning from Electrospun Nanofibres. Review Literature And Arts Of The Americas (2009).

9. Noruzi, M. Electrospun nanofibers in agriculture and food industry: A review. J. Sci. Food Agric. 96, 4663-4678 (2016).

10. Al-Zahrani, S. M. Utilization of polyethylene and paraffin waxes as controlled delivery systems for different fertilizers. Ind. Eng. Chem. Res. 39, 367-371 (2000).

11. Ni, B., Liu, M. \& Lu, S. Multifunctional slow-release urea fertilizer from ethylcellulose and superabsorbent coated formulations. Chem. Eng. J. 155, 892-898 (2009).

12. Chen, L., Xie, Z., Zhuang, X., Chen, X. \& Jing, X. Controlled release of urea encapsulated by starch-g-poly(l-lactide). Carbohydr. Polym. 72, 342-348 (2008).

13. Zhao, J. \& Wilkins, R. M. Low molecular weight polylactic acid as a matrix for the delayed release of pesticides. J. Agric. Food Chem. 53, 4076-4082 (2005).

14. Correia, D. M. et al. Influence of electrospinning parameters on poly ( hydroxybutyrate ) electrospun membranes fiber size and distribution. Polym. Eng. Sci. 54, 1608-1617 (2014).

15. Krishnamoorthy, V. \& Rajiv, S. An eco-friendly top down approach to nutrient incorporated electrospun seed coating for superior germination potential. J. Adv. Appl. Sci. Res. 1, 1-16 (2017).

16. Castro-Enriquez, D. D. et al. Preparation, characterization and release of urea from wheat gluten electrospun membranes. Materials (Basel). 5, 2903-2916 (2012).

17. Trenkel, M. E. Controlled-Release and Stabilized Fertilizers in Agriculture. Libro Fertilizantes (1997).

18. Kampeerapappun, P. \& Phanomkate, N. Slow release fertilizer from core-shell electrospun fibers. Chiang Mai J. Sci. 40, 775-782 (2013).

19. Ramakrishna, S., Fujihara, K., Teo, W., Lim, T. \& Ma, Z. An Introduction to Electrospinning and Nanofibers (World Scientific Publishing Company, Singapore, 2005).

20. Deitzel, J. M., Kleinmeyer, J., Harris, D. \& Beck Tan, N. C. The effect of processing variables on the morphology of electrospun nanofibers and textiles. Polymer (Guildf.) 42, 261-272 (2001).

21. Kenwright, A. M., Peace, S. K., Richards, R. W., Bunn, A. \& MacDonald, W. A. End group modification in poly(ethylene terephthalate). Polymer (Guildf). 40, 2035-2040 (1999).

22. Hobzov, R. et al. Morphological characterization of nanofibers : methods and application in practice. J. Nanomater. 2012, 1-14 (2012).

23. Zare, A., Morshed, M., Bagheri, R. \& Karimi, K. Effect of various parameters on the chemical grafting of amide monomers to poly (lactic acid). Fibers Polym. 14(11), 1783-1793 (2013).

24. Sadeghi-Avalshahr, A. R., Khorsand-Ghayeni, M., Nokhasteh, S., Molavi, A. M. \& Sadeghi-Avalshahr, M. Physical and mechanical characterization of PLLA interference screws produced by two stage injection molding method. Prog. Biomater. 5, 183-191 (2016).

25. Lu, P., Zhang, Y., Jia, C., Li, Y. \& Mao, Z. Use of polyurea from urea for coating of urea granules. Springerplus 5, 457 (2016).

26. Ahmad, S. I., Syed, I. A., Ravi Prasad, P. \& Ahmad, A. Quantitation of urea in urine by Fourier transforms infrared spectroscopy. Der Pharma Chem. 6, 90-96 (2014).

27. Colthup, N. B., Daly, L. H. \& Wiberley, S. E. Introduction to Infrared and Raman Spectroscopy (Academic Press, Cambridge, 1990).

28. Rošic, R. et al. The role of rheology of polymer solutions in predicting nanofiber formation by electrospinning. Eur. Polym. J. 48, 1374-1384 (2012).

\section{Acknowledgements}

This work was supported financially by the Centre for Crop Health at University of Southern Queensland (USQ), Australia. Authors thank Dr Peter Harris from Centre for Agricultural Engineering (CAE) at USQ for assistance in reviewing final versions of the paper. Authors acknowledge support from RMMF group at Royal Melbourne Institute of Technology University (RMIT) in using SEM (Scanning Electron Microscopy). We thank Nadia Zakhartchouk, technical coordinator at College of Science Engineering and Health, RMIT for assistance with BET analysis.

\section{Author contributions}

L.J. designed and performed the experiments, L.J. with T.L. analysed the data. L.J. wrote the manuscript with input from all authors. T.L., A.Y. and G.A. conceived the study and oversaw overall direction and planning.

\section{Competing interests}

The authors declare no competing interests. 


\section{Additional information}

Correspondence and requests for materials should be addressed to L.J.

Reprints and permissions information is available at www.nature.com/reprints.

Publisher's note Springer Nature remains neutral with regard to jurisdictional claims in published maps and institutional affiliations.

(c) (1) Open Access This article is licensed under a Creative Commons Attribution 4.0 International License, which permits use, sharing, adaptation, distribution and reproduction in any medium or format, as long as you give appropriate credit to the original author(s) and the source, provide a link to the Creative Commons licence, and indicate if changes were made. The images or other third party material in this article are included in the article's Creative Commons licence, unless indicated otherwise in a credit line to the material. If material is not included in the article's Creative Commons licence and your intended use is not permitted by statutory regulation or exceeds the permitted use, you will need to obtain permission directly from the copyright holder. To view a copy of this licence, visit http://creativecommons.org/licenses/by/4.0/.

(C) The Author(s) 2021 